\title{
Disease management interventions for improving self- management in lower-limb peripheral arterial disease (Protocol)
}

\author{
Inglis SC, Du H, Newton PJ, DiGiacomo M, Omari A, Davidson PM
}

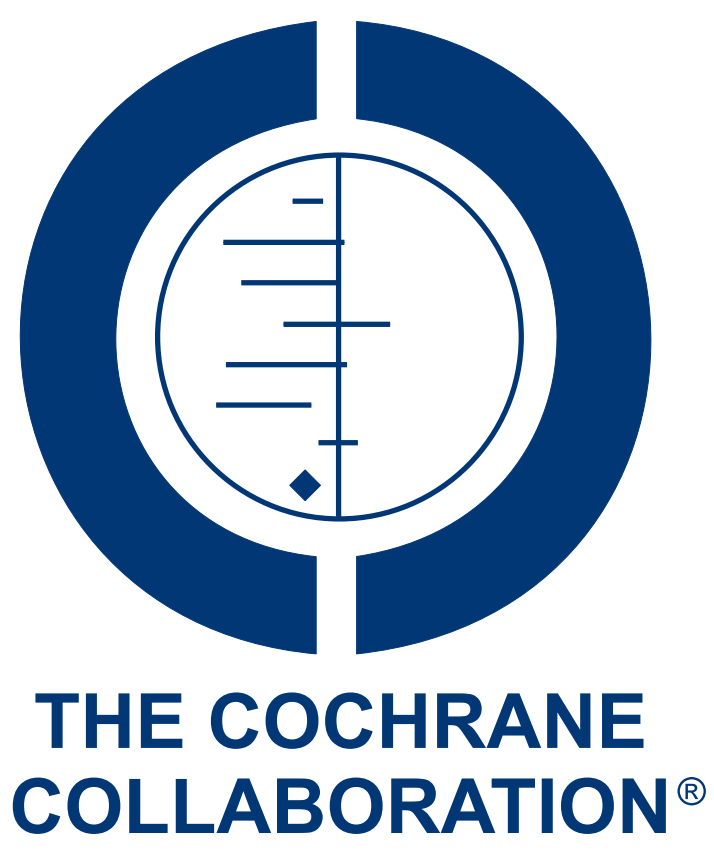

This is a reprint of a Cochrane protocol, prepared and maintained by The Cochrane Collaboration and published in The Cochrane Library 2012, Issue 3

http://www.thecochranelibrary.com

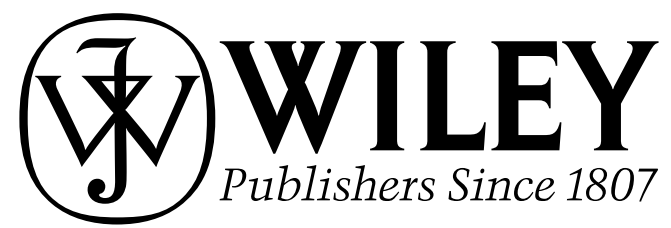

Disease management interventions for improving self-management in lower-limb peripheral arterial disease (Protocol)

Copyright (@) 2012 The Cochrane Collaboration. Published by John Wiley \& Sons, Ltd. 
TABLE OF CONTENTS

HEADER . . . . . . . . . . . . . . . . . . . . . . . . . . . . . . . . . . . . . . . . 1

ABSTRACT . . . . . . . . . . . . . . . . . . . . . . . . . . . . . . . . . . . . . . . . . . . . . . .

BACKGROUND . . . . . . . . . . . . . . . . . . . . . . . . . . . . . . . . . . . . 2

OBJECTIVES . . . . . . . . . . . . . . . . . . . . . . . . . . . . . . . . . . . . . . . . . . . . . .

METHODS . . . . . . . . . . . . . . . . . . . . . . . . . . . . . . . . . . . . . . 3

ACKNOWLEDGEMENTS . . . . . . . . . . . . . . . . . . . . . . . . . . . . . . . . . . . . . . . 6

REFERENCES . . . . . . . . . . . . . . . . . . . . . . . . . . . . . . . . . . . . . . . 47

APPENDICES . . . . . . . . . . . . . . . . . . . . . . . . . . . . . . . . . . . . . 9

HISTORY . . . . . . . . . . . . . . . . . . . . . . . . . . . . . . . . . . . . . . . 10

CONTRIBUTIONS OF AUTHORS . . . . . . . . . . . . . . . . . . . . . . . . . . . . . . . . . . . . . . 11

DECLARATIONS OF INTEREST . . . . . . . . . . . . . . . . . . . . . . . . . . . . . . . . . . . . . . . . 11

SOURCES OF SUPPORT . . . . . . . . . . . . . . . . . . . . . . . . . . . . . . . . . . 11

Disease management interventions for improving self-management in lower-limb peripheral arterial disease (Protocol)

Copyright $(2012$ The Cochrane Collaboration. Published by John Wiley \& Sons, Ltd. 


\title{
Disease management interventions for improving self- management in lower-limb peripheral arterial disease
}

\author{
Sally C Inglis ${ }^{1}$, Huiyun Du ${ }^{1}$, Phillip J Newton ${ }^{1}$, Michelle DiGiacomo ${ }^{1}$, Abdullah Omari ${ }^{2}$, Patricia M Davidson ${ }^{1}$ \\ ${ }^{1}$ Centre for Cardiovascular and Chronic Care, Faculty of Nursing, Midwifery and Health, University of Technology, Sydney, Sydney, \\ Australia. ${ }^{2}$ St Vincent's General Hospital, Sydney, Australia \\ Contact address: Sally C Inglis, Centre for Cardiovascular and Chronic Care, Faculty of Nursing, Midwifery and Health, University \\ of Technology, Sydney, Sydney, Australia. sally.inglis@uts.edu.au. \\ Editorial group: Cochrane Peripheral Vascular Diseases Group. \\ Publication status and date: New, published in Issue 3, 2012.
}

Citation: Inglis SC, Du H, Newton PJ, DiGiacomo M, Omari A, Davidson PM. Disease management interventions for improving self-management in lower-limb peripheral arterial disease. Cochrane Database of Systematic Reviews 2012, Issue 3. Art. No.: CD009714. DOI: $10.1002 / 14651858 . C D 009714$.

Copyright (C) 2012 The Cochrane Collaboration. Published by John Wiley \& Sons, Ltd.

\section{A B S T R A C T}

This is the protocol for a review and there is no abstract. The objectives are as follows:

The objective of this review is to systematically review, synthesise and quantify the effects of non-pharmacological and non-surgical chronic disease management interventions targeting self-management for people with lower-limb PAD. 


\section{B A C K G R O U N D}

\section{Description of the condition}

Peripheral arterial disease (PAD) is a chronic atherosclerotic cardiovascular disease impacting on quality of life and leading to adverse outcomes. The principal symptom of PAD is lower-limb pain or discomfort (known as intermittent claudication), which is typically brought on by walking and relieved by rest (Sutherland 2009). As PAD progresses, intermittent claudication may increase in severity and critical limb ischaemia may develop, which results in constant lower-limb pain at rest, leg ulceration, sepsis, and possibly gangrene and the need for amputation (Sutherland 2009). Several studies have indicated that the prevalence of PAD increases with age. A large study undertaken in San Diego, US identified a prevalence of $2.5 \%$ in people aged 60 years, $8.3 \%$ in those aged 60 to 69 years, and $18.8 \%$ in the study participants aged 70 years or older (Criqui 1985). Of 28,980 Scottish men and women screened using the ankle-brachial index (ABI), $10.9 \%$ of participants had an $\mathrm{ABI}$ which categorised them as 'at risk' (with an $\mathrm{ABI}<0.9$ indicating PAD and increased cardiovascular risk) (Price 2008). Another Scottish study identified that $4.6 \%$ of 2720 participants aged 55 to 74 years had intermittent claudication (lower-limb pain or discomfort brought on by walking and relieved by rest) and $8 \%$ had significant impairment of blood flow but were asymptomatic (Fowkes 1991).

Exercise, in particular regular walking, is a fundamental component of lower-limb PAD management (Hirsch 2005; Norgren 2007). Its effectiveness is proven by other Cochrane Reviews (Bendermacher 2006; Watson 2008). The latter showed that with exercise, including walking, skipping and running, the pain-free walking distance mean difference was 82.19 metres (95\% confidence interval (CI) 71.73 to 92.65 ) and the maximum walking distance mean difference was 113.20 metres (95\% CI 94.96 to 131.43) (Watson 2008). The benefits of exercise in PAD may be facilitated by the reversal of several pathological events that are common in PAD (for example arterial obstruction, endothelial dysfunction, altered skeletal muscle phenotype and inflammation) (Hamburg 2011). There are several important benefits of exercise for people with PAD. These are improved functional ability, cardiovascular fitness, physical mobility and psychological well being (Guidon 2010; Hamburg 2011). However, adhering to regular exercise can be problematic for people with PAD as the onset of intermittent claudication can act as a deterrent to exercise participation. A recent meta-analysis comparing endovascular treatment of intermittent claudication to supervised exercise reports that outcomes measured by treadmill walking were no different to the outcomes of endovascular treatment (Ahimastos 2011). The authors of the study concluded that "mechanisms that promote greater access to, uptake and sustainability of SVE (supervised exercise programs) would appear to be an important requirement to advance management of intermittent claudication given the favourable results" (Ahimastos 2011). Not only are access and uptake of such interventions important, but adherence is fundamental to the success of interventions such as exercise programs. Smoking is a substantial risk factor for the development (odds ratio $5.09,95 \%$ CI 2.97 to 8.72 ) (Leng 1995) and progression of lower-limb PAD and has significant implications for morbidity and mortality (Hirsch 2005; Norgren 2007). Quitting smoking is challenging and specialised support improves the success of attempts to quit (Fiore 2008). Like many other chronic diseases, people with PAD often experience poor health-related quality of life (Dumville 2004). There is evidence of socioeconomic disparity in the prevalence (Kroger 2009) and outcomes (Ferguson 2010) for people with PAD. Stigma and social marginalisation can be experienced by smokers (Stuber 2008).

Considering the psychological burden (Smolderen 2009), poor quality of life (Dumville 2004), risk of acute events (Criqui 2008) leading to increased morbidity and mortality, and management strategies requiring behavioural change and long-term adherence, chronic disease management interventions that aim to improve self-management may improve outcomes for people with PAD. Performance measures for management of PAD underscore the importance of risk assessment, patient education (in particular on the importance of exercise and smoking cessation), self-management (especially adherence to exercise and smoking cessation) and monitoring of disease status (Olin 2010). Based on this guideline, non-pharmacological, non-surgical disease management interventions for PAD should incorporate risk assessment, patient education, self-management strategies and monitoring of disease status.

\section{Description of the intervention}

Surgical and pharmacological interventions can provide symptom relief and reduce the risk of adverse outcomes for people with lower-limb PAD. However, benefits to quality of life and other outcomes can also be gained through non-surgical and non-pharmacological strategies, in particular, smoking cessation and exercise. However, adhering to exercise programmes and quitting smoking can be very challenging and interventions which promote and support the change of health behaviours and adherence to prescribed treatments may assist people with PAD to improve their own health. Health professionals typically refer to this concept as self-management. There is currently a lack of consensus on the definition of self-management (Gardetto 2011) and self-care is also often used as an interchangeable term. In this review, we will use the term self-management for consistency. Self-management is a core component of disease management interventions (Lovell 2011), which are organised programmes or services often led by a nurse or allied health professional. They aim to increase self-efficacy (a person's belief that they can achieve a given task) and knowledge about the disease, treatment and outcomes (health literacy) so that the person is better informed and capable of engaging in activities which protect or promote their own health 
(for example exercise participation and quitting smoking), manage their symptoms (for example managing intermittent claudication through medications and pain management strategies), the impact which lower-limb PAD has on their life, and also adhere to any prescribed therapies (such as a prescribed exercise regime or risk-reducing medications). These interventions may be delivered face-to-face (in a group or one-on-one session), via telephone or other information communication technologies, such as Internet, short message service (SMS) or e-mail, and will often involve a combination of delivery modes. Increasingly the role of disease management is emphasised to address the burden of chronic conditions, and self-management is a core component of disease management. Lorig 1993 defines self-management approaches as helping individuals "to make informed choices, to adapt new perspectives and generic skills that can be applied to new problems as they arise, to practise new health behaviours, and to maintain or regain emotional stability”. Gardetto 2011 has combined definitions of self-management by Lorig 2003 and Barlow 2002, defining selfmanagement as "the individual's ability (problem solving, decision making, resource utilization, formation of patient-provider partnerships, action planning, and tailoring of daily activities) to undertake and manage day-to-day tasks, inherent lifestyle changes, physical symptoms, and psychosocial consequences of health and well-being over the lifetime of an illness". These definitions emphasise the importance of a patient-provider partnership where information is tailored and targeted to the needs of an individual and action planning and self-monitoring are integral to achieving optimal health outcomes. Like in many other chronic conditions, the role of disease management interventions in PAD is to reduce symptoms and improve clinical outcomes (Lovell 2011).

\section{How the intervention might work}

For many chronic conditions, adhering to both pharmacological and non-pharmacological interventions is important to improve survival, functional status and health-related quality of life. Adherence with these recommendations also improves organisational outcomes such as decreasing admissions to hospital. Promoting and enhancing self-management strategies is an integral element of chronic disease management programs which have a strong evidence base across many conditions, such as heart failure (Inglis 2010), chronic obstructive respiratory disease (Effing 2007), osteoarthritis and diabetes (Deakin 2005). These approaches recognise the need to shift the focus on purely acute events to a focus on supporting the individual living with a chronic condition. Incorporating critical elements such as patient empowerment, increasing adherence to evidence-based recommendations and promoting care coordination can improve health outcomes (Tsai 2005). Increasingly, health providers recognise that information alone is not sufficient to promote adherence and drive behaviour change. This has led to multiple interventions to assist individuals in adopting favourable health behaviours and to engage in self-management strategies. Systematic reviews of integrated disease management strategies have shown that coordinated strategies and promoting self-management can improve health outcomes, yet identifying the key components of interventions is less clear (Coster 2009). Chronic disease management interventions which are designed to improve self-management typically focus on increasing self-efficacy, health literacy, symptom monitoring and management, and adherence. By addressing these important aspects of managing and living with a chronic disease, these interventions can also improve outcomes and health-related quality of life. Adherence to exercise regimes, smoking cessation and risk reducing pharmacotherapies is important for managing PAD. Making a change to health behaviours can be very challenging, especially if this involves a lifetime habit such as smoking or exercise which causes pain and discomfort. These interventions might work by engaging people with PAD in decisions regarding their own health as well as by using behavioural change strategies to promote smoking cessation, exercise participation and other lifestyle modifications.

\section{Why it is important to do this review}

Evidence supports the use of chronic disease management interventions to improve self-management in chronic diseases other than PAD (Deakin 2005; Effing 2007; Inglis 2010), however it is unclear what benefits these interventions offer for people with PAD. To our knowledge, there are no other systematic reviews of the evidence for chronic disease management interventions to improve self-management for lower-limb PAD.

\section{O B J E C T I VES}

The objective of this review is to systematically review, synthesise and quantify the effects of non-pharmacological and non-surgical chronic disease management interventions targeting self-management for people with lower-limb PAD.

\section{METHODS}

\section{Criteria for considering studies for this review}

\section{Types of studies}

We will include randomised controlled trials (RCTs) of chronic disease management interventions to support self-management for people with lower-limb PAD. 


\section{Types of participants}

Adults (aged $\geq 16$ years) of either sex, any age or ethnic group with a diagnosis of lower-limb PAD, ideally defined by the ankle-brachial index $(\mathrm{ABI})$ measurement $(\mathrm{ABI}<0.9)$ or diagnostic equivalent. We will include studies which do not report an $\mathrm{ABI}$ or diagnostic equivalent to confirm diagnosis of PAD but state that participants were patients with PAD or had intermittent claudication. Study participants may be from hospital or community cohorts. We will exclude studies dealing with general cardiac or vascular disorders rather than specifically with PAD unless the intervention is tailored to individual patient needs (that is addresses the needs specific to people with PAD and involves the key characteristics of self-management) and data are available for participants with PAD separate from participants with other cardiac or vascular disorders.

\section{Types of interventions}

We will include chronic disease management interventions designed to engage people with lower-limb PAD in activities that protect and promote their own health (for example smoking cessation, diet, exercise). These interventions must have a component that supports people in understanding, monitoring and managing symptoms of lower-limb PAD and the impact of lower-limb PAD has on their life (intermittent claudication) as well as maintaining lifestyle modifications that are recommended to people with PAD and adherence to prescribed therapies.

Interventions which involve exercise or everyday physical activity, or both, will be considered for inclusion. However, in addition to prescribing or facilitating an exercise regime, the intervention must be multifaceted and include additional components such as support in understanding, monitoring and managing symptoms of lower-limb PAD and the impact lower-limb PAD has on their life (intermittent claudication), as well as maintaining lifestyle modifications that are recommended to people with PAD and adherence to prescribed therapies. Interventions with no self-management component will be excluded. These interventions will include an active component of support or interaction with the patient as opposed to the mere provision of recommendations about therapies to patients. Education interventions should include some form of assessment of understanding and follow-up.

These interventions could take the form of, but are not limited to, interactive education, telemonitoring, self-monitoring, a peer-support group, coaching and motivational interviewing (Lai 2010). Modifiable risk factors such as smoking and adherence to exercise can be addressed with behaviour change strategies that consider the individuals' beliefs, values and motivation to change. We will not exclude studies based on the personnel delivering the intervention and our review may include programmes delivered by lay personnel (Foster 2007).

We will compare these interventions with usual care, defined as no specialist chronic disease management intervention aimed at im- proving self-management. For example, usual care could involve a patient receiving instructions from a clinician to stop smoking and commence an exercise programme, but the patient is not enrolled in a programme which addresses self-management specifically and there is no ongoing follow-up of the patient to encourage or support self-management. Interventions will not be performed as part of a trial of surgical or pharmacological interventions and at the same time. The only difference between the intervention and control arms in terms of care received should be the chronic disease management intervention to support self-management.

\section{Types of outcome measures}

We will not exclude studies based on the outcomes reported. We will examine immediate outcomes (up to six weeks from the commencement of the intervention), intermediate (up to and including one year from the commencement of the intervention), and longer-term outcomes (measured more than one year after the intervention).

\section{Primary outcomes}

We will examine the following as our primary outcomes.

- Functional status will be examined in separate meta-analyses according to the individual measures of distance, such as:

o absolute claudication distance;

- initial claudication distance;

$\circ$ the six-minute walk test.

- Functional status will also be examined according to the individual questionnaires, such as: 1994);

- Summary Lower Extremity Performance Score (Vogt

○ Walking Impairment Questionnaire (Regensteiner 1990);

- Baltimore Activity Scale (Gardner 2006).

- Health-related quality of life measured using PAD-specific validated questionnaires, such as the Intermittent Claudication Questionnaire (Chong 2002), the Claudication Scale (CLAU-S) (Spengel 1997); or generic health-related quality of life measures such as the Medical Outcomes Short-Form (SF) 36 (Ware 1992) and its related instruments or the EuroQoL (EQ-5D) (The EuroQol Group 1990).

\section{Secondary outcomes}

We will examine the following as our secondary outcomes:

- all-cause mortality;

- revascularisation or amputation;

- acute events (stroke or myocardial infarction);

- self-efficacy for exercise (using validated measures such as Bandura’s Self-Efficacy for Exercise Scale (Bandura 1994));

- modifiable risk factors (smoking, obesity, blood pressure, lipids); 
diet)

- adherence to prescribed therapies (exercise, medications,

- patient acceptance and satisfaction with the intervention;

- adverse events.

Adverse events are not a commonly reported outcome in nonpharmacological, non-surgical chronic disease management studies, however improved adherence to pharmacological interventions may lead to increases in adverse event rates and hence these will be examined, if reported.

\section{Search methods for identification of studies}

We will not apply date limits to database searches. We will search all databases from commencement of the database up to the search date. Language restrictions will not apply to any of the searches.

\section{Electronic searches}

The Cochrane Peripheral Vascular Diseases Group Trials Search Co-ordinator (TSC) will search the Specialised Register and the Cochrane Central Register of Controlled Trials (CENTRAL), part of The Cochrane Library (www.the cochranelibrary.com). See Appendix 1 for details of the search strategy which will be used to search CENTRAL. The Specialised Register is maintained by the TSC and is constructed from weekly electronic searches of MEDLINE, EMBASE, CINAHL, AMED, and through handsearching relevant journals. The full list of the databases, journals and conference proceedings which have been searched, as well as the search strategies used, are described in the Specialised Register section of the Cochrane Peripheral Vascular Diseases Group module in The Cochrane Library (www.thecochranelibrary.com).

In addition, the authors will search the following databases using search strategies based on the CENTRAL search strategy.

- PsycINFO.

- Web of Science.

- ProQuest Dissertations \& Theses Database.

- ANU Digital Theses Collection.

- Index to Theses.

\section{Searching other resources}

We will review bibliographies of retrieved studies to identify other relevant studies. We will use citation tracking to identify other publications related to the included studies or other relevant references.

In addition, we will search our personal literature collections for relevant studies.

We will handsearch conference abstracts to identify relevant abstracts of randomised controlled trials of self-management interventions for people with PAD. Due to resource constraints, we will handsearch abstracts for the following conferences for the past five to 10 years if we are able to obtain copies of abstract books or access to online resources:

- American Heart Association Annual Scientific Sessions;

- Society for Vascular Surgery Annual Meetings;

- Society for Vascular Nursing Annual Conventions;

- European Society for Vascular Surgery Annual Meetings.

\section{Data collection and analysis}

Two review authors will review all identified abstracts and results from database searches for relevance to the review topic. If the reference appears relevant, we will obtain a full copy of the reference for detailed review to determine the inclusion in the review or exclusion of the study.

\section{Selection of studies}

Two review authors will independently review the results of each search according to the exclusion and inclusion criteria. A third review author will adjudicate in the instance of disagreement between the first two review authors.

\section{Data extraction and management}

Two review authors will extract data from the included studies in a blinded manner and a third review author will check all extracted data for accuracy and consistency. We will use a customised electronic data extraction form to record all extracted data.

\section{Assessment of risk of bias in included studies}

Two review authors will independently assess risk of bias (methodological quality). A third review author will adjudicate in the instance of disagreement between the first two review authors. We will assess the risk of bias in the following domains using the Cochrane Collaboration tool (Higgins 2011):

- sequence generation;

- allocation concealment;

- blinding;

- incomplete outcome data;

- selective outcome reporting;

- other sources of bias.

We will rate the assessment of risk of bias as 'unclear risk of bias', 'low risk of bias' and 'high risk of bias' according to the Cochrane Collaboration tool (Higgins 2011) for assessing risk of bias; examples from the text will be provided to support this classification.

\section{Measures of treatment effect}

We will analyse outcomes which are continuous variables using difference in means and the $95 \%$ confidence intervals (CI) for studies using the same outcome measurement scale. In the instance of 
studies reporting the same outcome (for example quality of life or measures of functional status) but using different measurements, we will use the standardised mean difference and $95 \%$ confidence interval to report these data.

We will report risk ratios (RR) for outcomes which are dichotomous variables (that is all-cause mortality, revascularisation or amputation, and acute events).

\section{Unit of analysis issues}

If the included trials report outcomes at more than one time point, we will report the primary outcome data. That is, the main time point used in the analysis in the primary publication of the study.

\section{Dealing with missing data}

We will contact study authors via e-mail in the instance of missing or unclear data in order to maximise data synthesis. Where possible, we will perform all analyses using intention-to-treat analysis, that is, we will analyse all participants and their outcomes in the groups to which they were allocated regardless of whether they received the intervention or whether or not they were assessed for the outcome. In the event of dropouts, we will adjust the denominator for the number of participants followed up for each outcome if these data are available.

\section{Assessment of heterogeneity}

We will assess statistical heterogeneity for the outcomes metaanalysed using the $\mathrm{I}^{2}$ statistic, which describes the percentage of variability in effect estimates due to heterogeneity (Deeks 2011).

\section{Assessment of reporting biases}

Funnel plots allow review authors to make a visual assessment of whether small-study effects may be present in a meta-analysis (Sterne 2011). Small-study effects, when the intervention effect is more beneficial in smaller studies, is considered to be possibly caused by publication bias. We will explore this potential for smallstudy effects in the primary outcomes of the review further using funnel plots if at least 10 studies are included in the meta-analysis of the primary outcomes (Sterne 2011).

\section{Data synthesis}

We will quantitatively meta-analyse primary outcomes and some secondary outcomes (all-cause mortality, revascularisation, amputation, stroke or myocardial infarction, smoking status) depending on the availability of suitable data from high-quality studies. Considering the likely heterogeneity in participant populations, intervention characteristics, length of follow-up and outcome measurement, we will perform a random-effects meta-analysis.

We will tabulate and describe other outcomes such as adherence, self-efficacy for exercise, modifiable risk factors (obesity, blood pressure, lipids) and patient acceptance and satisfaction with the intervention.

We will include the following outcomes in the summary of findings table: functional status, health-related quality of life, all-cause mortality, revascularisation, amputation and smoking status.

\section{Subgroup analysis and investigation of heterogeneity}

If the number of included studies permits, we will perform subgroup analyses and investigations of heterogeneity for each outcome meta-analysed by considering each particular type of intervention. For example, some studies may use a face-to-face in a group or one-on-one format, other studies may include an Internet, e-mail, telephone-based or SMS delivered intervention. We will also consider the elements of interventions, that is clinician delivered, theoretically derived, and the intensity of the intervention, if the number of included studies permits. If the number of studies permits, we will also perform subgroup analyses to examine the clinical characteristics of the study participants, such as history of revascularisation.

\section{Sensitivity analysis}

We will perform sensitivity analyses if issues with methodological quality of the included studies are identified. Additionally, we will perform a sensitivity analysis to assess the influence of publication type (full peer-reviewed publication versus abstract or thesis) on reported outcomes. We will perform further sensitivity analyses to assess the effect of dropouts on outcomes, that is, in the event of dropouts we will not adjust the denominator for the number of participants followed up for each outcome and the denominator will be the total number of participants randomised to each arm irrespective of whether or not they were followed up for the outcome. If a sensitivity analysis indicates the results are influenced by particular methodological or publication factors, we will discuss the findings of the review within this context.

\section{ACKNOWLEDGEMENTS}

Dr Sally C Inglis is a Post-Doctoral Research Fellow supported by the National Health and Medical Research Council of Australia (NHMRC) and National Heart Foundation of Australia (NHMRC Grant ID 472 699).

Dr Phillip J Newton is a Post-Doctoral Research Fellow supported by a Chancellor's Post-Doctoral Fellowship from the University of Technology, Sydney.

Dr Michelle DiGiacomo is a Post-Doctoral Research Fellow supported by University of Technology, Sydney, Curtin University and the NHMRC (NHMRC Grant ID 533 547). 


\section{R E F E R E N C E S}

\section{Additional references}

Ahimastos 2011

Ahimastos AA, Pappas EP, Buttner PG, Walker PJ,

Kingwell BA, Golledge J. A meta-analysis of the outcome of endovascular and non-invasive therapies in the treatment of intermittent claudication. Journal of Vascular Surgery 2011; 54(5):1511-21.

\section{Bandura 1994}

Bandura A. Self-efficacy. In: Ramachaudran V editor (s). Encyclopedia of Human Behavior. Vol. 4, New York: Academic Press, 1994:71-81.

\section{Barlow 2002}

Barlow J, Wright C, Sheasby J, Turner A, Hainsworth J. Self-management approaches for people with chronic conditions: a review. Patient Education and Counseling 2002;48(2):177-87.

\section{Bendermacher 2006}

Bendermacher BL, Willigendael EM, Teijink JA, Prins MH. Supervised exercise therapy versus non-supervised exercise therapy for intermittent claudication. Cochrane Database of Systematic Reviews 2006, Issue 2. [DOI: 10.1002/ 14651858.CD005263.pub2]

\section{Chong 2002}

Chong PF, Garratt AM, Golledge J, Greenhalgh RM, Davies AH. The Intermittent Claudication Questionnaire: A patient-assessed condition-specific health outcome measure. Journal of Vascular Surgery 2002;36(4):764-71.

\section{Coster 2009}

Coster S, Norman I. Cochrane reviews of educational and self-management interventions to guide nursing practice: a review. International Journal of Nursing Studies 2009;46(4): 508-28.

\section{Criqui 1985}

Criqui MH, Fronek A, Barrett-Connor E, Klauber MR, Gabriel S, Goodman D. The prevalence of peripheral arterial disease in a defined population. Circulation 1985; 71:510-5.

\section{Criqui 2008}

Criqui MH, Ninomiya JK, Wingard DL, Ji M, Fronek A. Progression of peripheral arterial disease predicts cardiovascular disease morbidity and mortality. Journal of the American College of Cardiology 2008;52:1736-42.

\section{Deakin 2005}

Deakin TA, McShane CE, Cade JE, Williams R. Group based training for self-management strategies in people with type 2 diabetes mellitus. Cochrane Database of Systematic Reviews 2005, Issue 2. [DOI: 10.1002/ 14651858.CD003417.pub2]

\section{Deeks 201}

Deeks JJ, Higgins JPT, Altman DG. Chapter 9: Analysing data and undertaking meta-analyses. In: Higgins JPT, Green S, editors. Cochrane Handbook for Systematic Reviews of Interventions Version 5.1.0 [updated March
2011]. The Cochrane Collaboration, 2011. Available from www.cochrane-handbook.org.

\section{Dumville 2004}

Dumville JC, Lee AJ, Smith FB, Fowkes FG. The healthrelated quality of life of people with peripheral arterial disease in the community: the Edinburgh Artery Study. British Journal of General Practice 2004;54(508):826-31.

\section{Effing 2007}

Effing T, Monninkhof EEM, van der Valk PP, Zielhuis GGA, Walters EH, van der Palen JJ, et al.Self-management education for patients with chronic obstructive pulmonary disease. Cochrane Database of Systematic Reviews 2007, Issue 4. [DOI: 10.1002/14651858.CD002990.pub2]

\section{Ferguson 2010}

Ferguson HJ, Nightingale P, Pathak R, Jayatunga AP. The Influence of socio-economic deprivation on rates of major lower limb amputation secondary to peripheral arterial disease. European Journal of Vascular and Endovascular Surgery 2010;40(1):76-80.

Fiore 2008

Fiore FC, Jaén CR, Baker TB, Bailey WC, Benowitz NL, Curry SJ, et al.Treating Tobacco Use and Dependence: 2008 Update. Clinical practice guideline. Rockville MD: U.S. Department of Health and Human Services Public Health Service, 2008.

\section{Foster 2007}

Foster G, Taylor SJC, Eldridge S, Ramsay J, Griffiths CJ. Self-management education programmes by lay leaders for people with chronic conditions. Cochrane Database of Systematic Reviews 2007, Issue 4. [DOI: 10.1002/ 14651858.CD005108.pub2]

\section{Fowkes 1991}

Fowkes FGR, Housely E, Cawood EHH, MacIntyre CCA, Ruckley CV, Prescott RJ. Edinburgh Artery Study: prevalence of asymptomatic and symptomatic peripheral arterial disease in the general population. International Journal of Epidemiology 1991;20(2):384-92.

\section{Gardetto 2011}

Gardetto NJ. Self-management in heart failure: where have we been and where should we go?. Journal of Multidisciplinary Healthcare 2011;4:39-51.

\section{Gardner 2006}

Gardner AW, Montgomery PS. The Baltimore Activity Scale for intermittent claudication:a validation study. Vascular and Endovascular Surgery 2006;40(5):383-91.

\section{Guidon 2010}

Guidon M, McGee H. Exercise-based interventions and health-related quality of life in intermittent claudication: A 20-year (1989-2008) review. European Journal of Cardiovascular Prevention and Rehabilitation 2010;17(2): 140-54. 


\section{Hamburg 2011}

Hamburg NM, Balady GJ. Exercise rehabilitation in peripheral artery disease functional impact and mechanisms of benefits. Circulation 2011;123:87-97.

\section{Higgins 2011}

Higgins JPT, Altman DG, Sterne JAC. Chapter 8: Assessing risk of bias in included studies. In: Higgins JPT, Green $S$, editors. Cochrane Handbook for Systematic Reviews of Interventions Version 5.1.0 [updated March 2011]. The Cochrane Collaboration, 2011. Available from www.cochrane-handbook.org.

\section{Hirsch 2005}

Hirsch AT, Haskal ZJ, Hertzer NR, Bakal CW, Creager MA, Halperin JL, et al.ACC/AHA 2005 Practice Guidelines for the Management of Patients With Peripheral Arterial Disease (Lower Extremity, Renal, Mesenteric, and Abdominal Aortic): A Collaborative Report from the American Association for Vascular Surgery/Society for Vascular Surgery, ${ }^{*}$ Society for Cardiovascular Angiography and Interventions, Society for Vascular Medicine and Biology, Society of Interventional Radiology, and the ACC/AHA Task Force on Practice Guidelines (Writing Committee to Develop Guidelines for the Management of Patients With Peripheral Arterial Disease): Endorsed by the American Association of Cardiovascular and Pulmonary Rehabilitation; National Heart, Lung, and Blood Institute; Society for Vascular Nursing; TransAtlantic Inter-Society Consensus; and Vascular Disease Foundation. Circulation 2006;113:e463-e5

\section{Inglis 2010}

Inglis SC, Clark RA, McAlister FA, Ball J, Lewinter C, Cullington D, et al.Structured telephone support or telemonitoring programmes for patients with chronic heart failure. Cochrane Database of Systematic Reviews 2010, Issue 8. [DOI: 10.1002/14651858.CD007228.pub2]

\section{Kroger 2009}

Kroger K, Dragano N, Stang A, Moebus S, Mohlenkamp S, Mann K, et al.An unequal social distribution of peripheral arterial disease and the possible explanations: results from a population-based study. Vascular Medicine 2009;14(4): 289-96.

Lai 2010

Lai DTC, Cahill K, Qin Y, Tang JL. Motivational interviewing for smoking cessation. Cochrane Database of Systematic Reviews 2010, Issue 1. [DOI: 10.1002/ 14651858.CD006936.pub2]

\section{Leng 1995}

Leng GC, Lee AJ, Fowkes FG, Lowe GD, Housley E. The relationship between cigarette smoking and cardiovascular risk factors in peripheral arterial disease compared with ischaemic heart disease. The Edinburgh Artery Study. European Heart Journal 1995;16(11):1542-8.

\section{Lorig 1993}

Lorig K. Self-management of chronic illness: a model for the future. Generations 1993;17(3):11-4.
Lorig 2003

Lorig KR, Holman HR. Self-management education: history, definition, outcomes and mechanisms. Annals of Behavioral Medicine 2003;26(1):1-7.

\section{Lovell 2011}

Lovell M, Myers K, Forbes TL, Dresser G, Weiss E. Peripheral arterial disease: application of the chronic care model. Journal of Vascular Nursing 2011;29(4):147-52.

\section{Norgren 2007}

Norgren L, Hiatt WR, Dormandy JA, Nehler MR, Harris KA, Fowkes FGR. Inter-Society Consensus for the Management of Peripheral Arterial Disease (TASC II). European Journal of Vascular and Endovascular Surgery 2007; 33 Suppl:1-70.

Olin 2010

Olin JW, Allie DE, Belkin M, Bonow RO, Casey DE Jr, Creager MA, et al.ACCF/AHA/ACR/SCAI/SIR/SVM/ SVN/SVS 2010 Performance Measures for Adults With Peripheral Artery Disease: A Report of the American College of Cardiology Foundation/American Heart Association Task Force on Performance Measures, the American College of Radiology, the Society for Cardiac Angiography and Interventions, the Society for Interventional Radiology, the Society for Vascular Medicine, the Society for Vascular Nursing, and the Society for Vascular Surgery (Writing Committee to Develop Clinical Performance Measures for Peripheral Artery Disease) Developed in Collaboration With the American Association of Cardiovascular and Pulmonary Rehabilitation; the American Diabetes Association; the Society for Atherosclerosis Imaging and Prevention; the Society for Cardiovascular Magnetic Resonance; the Society of Cardiovascular Computed Tomography; and the PAD Coalition Endorsed by the American Academy of Podiatric Practice Management. Journal of the American College of Cardiology 2010;56:2147-81.

\section{Price 2008}

Price JF, Stewart MCW, Douglas AF, Murray GD, Fowkes GFR. Frequency of a low ankle brachial index in the general population by age, sex and deprivation: cross-sectional survey of 28980 men and women. European Journal of Cardiovascular Prevention and Rehabilitation 2008;15(3): 370-5.

\section{Regensteiner 1990}

Regensteiner JG, Steiner JF, Panzer RJ, Hiatt WR. Evaluation of walking impairment by questionnaire in patients with peripheral arterial disease. Journal of Vascular Medicine and Biology 1990;2:142-52.

\section{Smolderen 2009}

Smolderen KG, Hoeks SE, Pedersen SS, van Domburg RT, de Liefde II, Poldermans D. Lower-leg symptoms in peripheral arterial disease are associated with anxiety, depression, and anhedonia. Vascular Medicine 2009; 14: 297-304.

\section{Spengel 1997}

Spengel FA, Brown TM, Dietze S, Kirchberger I, Comte S. The claudication scale (CLAU-S): a new disease-specific 
quality of life instrument in intermittent claudication. Disease Management and Health Outcomes 1997;2 Suppl 1: 65-70

\section{Sterne 2011}

Sterne JAC, Egger M, Moher D. Chapter 10: Addressing reporting biases. In: Higgins JPT, Green S, editors. Cochrane Handbook for Systematic Reviews of Interventions. Version 5.1.0 [updated March 2011]. The Cochrane Collaboration, 2011. Available from www.cochrane-handbook.org.

\section{Stuber 2008}

Stuber J, Galea S, Link BG. Smoking and the emergence of a stigmatized social status. Social Science and Medicine 2008;67(3):420-30.

\section{Sutherland 2009}

Sutherland JA, Leya FS, Dieter RS. Chapter 16: History and physical examination. In: Dieter RS, Dieter RA Jr, Dieter RA 3rd editor(s). Peripheral arterial disease. New York: McGraw Hill, 2009:250-67.

\section{The EuroQol Group 1990}

The EuroQol Group. EuroQol-a new facility for the measurement of health-related quality of life. Health Policy
1990;16(3):199-208.

\section{Tsai 2005}

Tsai AC, Morton SC, Mangione CM, Keeler EB. A metaanalysis of interventions to improve care for chronic illnesses. The American Journal of Managed Care 2005;11 (8):478-88.

Vogt 1994

Vogt MT, Cauley JA, Kuller LH, Nevitt MC. Functional status and mobility among elderly women with lower extremity arterial disease: the study of osteoporotic fractures. Journal of the American Geriatric Society 1994;42 (9):923-9.

\section{Ware 1992}

Ware JE Jr, Sherbourne CD. The MOS 36-item short-form health survey (SF-36) . I. Conceptual framework and item selection. Medical Care 1992;30(6):473-83.

\section{Watson 2008}

Watson L, Ellis B, Leng GC. Exercise for intermittent claudication. Cochrane Database of Systematic Reviews 2008, Issue 4. [DOI: 10.1002/14651858.CD000990.pub2]

* Indicates the major publication for the study

\section{A P P E N D I CES}

\section{Appendix I. CENTRAL search strategy}

\#1 MeSH descriptor Arteriosclerosis, this term only \#2 MeSH descriptor Arteriolosclerosis, this term only \#3 MeSH descriptor Arteriosclerosis Obliterans, this term only \#4 MeSH descriptor Atherosclerosis, this term only \#5 MeSH descriptor Arterial Occlusive Diseases, this term only \#6 MeSH descriptor Intermittent Claudication, this term only \#7 MeSH descriptor Ischemia, this term only \#8 MeSH descriptor Peripheral Vascular Diseases explode all trees \#9 (arter* or vascular or vein* or veno* or peripher*) near (occlus* or reocclus* or re-occlus* or steno* or obstuct* ${ }^{*}{ }^{*}{ }^{*}$ lesio* or block* $^{*}$ or harden* or stiffen*)

\#10 (peripheral near3 dis $^{*}$ )

\#11 claudic* or IC

\#12 (isch* or CLI)

\#13 (leg or limb) near4 (obstruct* or occlus* or steno*)

\#14 dysvascular*

\#15 (\#1 OR \#2 OR \#3 OR \#4 OR \#5 OR \#6 OR \#7 OR \#8 OR \#9 OR \#10 OR \#11 OR \#12 OR \#13 OR \#14) \#16 MeSH descriptor Self Care explode all trees

\#17 self near3 (care or help or manag* or directed or monitor* or efficacy or admin*)

\#18 symptom near3 (care or help or manag* or directed or monitor* or efficacy or admin*)

\#19 MeSH descriptor Patient Education as Topic, this term only

\#20 (patient near3 (education or promot*))

\#21 (patient near3 (information or care or manage*)) 
\#22 (health near3 (education or promot $^{*}$ ))

\#23 health near3 information

\#24 empower*

\#25 MeSH descriptor Adaptation, Psychological explode all trees

\#26 psychologic* near3 adjust*

\#27 psychologic* near3 adapt*

\#28 cope or copes or coping

\#29 adapt* near4 behav*

\#30 behav* near4 therap*

\#31 behav* near4 intervent*

\#32 MeSH descriptor Counseling explode all trees

\#33 counsel* $^{*}$ or feedback

\#34 MeSH descriptor Health Education explode all trees

\#35 health near3 literacy

\#36 health near3 educt*

\#37 MeSH descriptor Motivation, this term only

\#38 motivat*

\#39 MeSH descriptor Cognitive Therapy, this term only

\#40 MeSH descriptor Psychotherapy explode all trees

\#41 psychoeducation

\#42 psycho-education

\#43 psychotherap*

\#44 psycho-therap*

\#45 MeSH descriptor Attitude to Health explode all trees

\#46 MeSH descriptor Disease Management, this term only

\#47 (disease near3 manag*):ti,ab,kw

\#48 MeSH descriptor Managed Care Programs, this term only

\#49 (manage* near3 care):ti,ab,kw

\#50 MeSH descriptor Patient Care Planning explode all trees

\#51 (care near3 (patient or nursing or goal)):ti,ab,kw

\#52 MeSH descriptor Delivery of Health Care, Integrated explode all trees

\#53 (integrated near3 (health or care* or system or delivery)):ti,ab, kw

\#54 ((interdisciplin* or multidisciplin*) near3 (care or health or deliver* or system)):ti,ab,kw

\#55 MeSH descriptor Patient Care Management, this term only

\#56 MeSH descriptor Patient-Centered Care, this term only

\#57 MeSH descriptor Reminder Systems, this term only

\#58 (reminder near3 system*):ti,ab,kw

\#59 (peer near3 support):ti,ab,kw

\#60 (coach*):ti,ab,kw

\#61 MeSH descriptor Telemedicine, this term only

\#62 (telemedicine or telemonitor*):ti,ab,kw

\#63 (self near3 (care or monitor*)):ti,ab,kw

\#64 (care near3 plan):ti,ab,kw

\#65 \#16 OR \#17 OR \#18 OR \#19 OR \#20 OR \#21 OR \#22 OR \#23 OR \#24 OR \#25 OR \#26 OR \#27 OR \#28 OR \#29 OR \#30

OR \#31 OR \#32 OR \#33 OR \#34 OR \#35 OR \#36 OR \#37 OR \#38 OR \#39 OR \#40 OR \#41 OR \#42 OR \#43 OR \#44 OR \#45

OR \#46 OR \#47 OR \#48 OR \#49 OR \#50 OR\#51 OR \#52 OR \#53 OR \#54 OR \#55 OR \#56 OR \#57 OR \#58 OR \#59 OR \#60

OR \#61 OR \#62 OR \#63 OR \#64)

\#66 (\#15 AND \#65) 


\section{H I S T O R Y}

Protocol first published: Issue 3, 2012

\section{CONTRIBUTIONS OFAUTHORS}

Sally C Inglis: responsible for conception and design of this review. Responsible for writing the protocol. Reviewed and revised the protocol for intellectual content.

Huiyun Du: reviewed and revised the protocol for intellectual content.

Phillip J Newton: reviewed and revised the protocol for intellectual content.

Michelle DiGiacomo: reviewed and revised the protocol for intellectual content.

Abdullah Omari: reviewed the protocol for intellectual content.

Patricia M Davidson: reviewed and revised the protocol for intellectual content.

\section{DECLARATIONS OF INTEREST}

None known

\section{SOURCES OF SUPPORT}

\section{Internal sources}

- No sources of support supplied

\section{External sources}

- Chief Scientist Office, Scottish Government Health Directorates, The Scottish Government, UK. The PVD Group editorial base is supported by the Chief Scientist Office. 